\title{
La pedagogía de proyectos en la escuela: una aproximación a sus discursos en el caso del área de lenguaje
}

Nylza Offir García Vera'

nogarcia@pedagogica.edu.co, nylzao@yahoo.es

\section{Resumen}

El artículo analiza los discursos que fundamentan el enfoque de la pedagogía de proyectos y su consecuente desarrollo y aplicación en el campo de la didáctica de la lengua en Colombia, destacando la pluralidad de versiones y materializaciones pedagógicas que se hacen del mismo. Haciendo un rastreo histórico a las primeras fuentes y referencias del enfoque conocido como "método de proyectos"y pasando por la descripción de algunos planteamientos más recientes, se destacan dos supuestos de base que conforman actualmente dicho discurso en la educación: el pragmatismo y el constructivismo.

\section{Palabras clave}

Pedagogía de proyectos, didáctica de la lengua, pragmatismo, constructivismo.

\section{ABstract}

The article analyzes the theoretical tenets that substantiate the pedagogy of projects' approach and its consequent development and application in the field of the didactics of language in Colombia. Likewise, the plurality of versions and pedagogic materializations that have been made of it are highlighted. Following the first sources and references of the approach known as "method of projects" and describing some more recent positions, two base suppositions that found this discourse at the moment in the education have stood out: pragmatism and constructivism.

\section{KEYWORDS}

Pedagogy of projects, didactics of language, pragmatism, constructivism.

\footnotetext{
1 Profesora de la Facultad de Educación, Universidad Pedagógica Nacional. Candidata a Magíster en Pedagogía, especialista en enseñanza del español y la literatura.
} 


\section{Introducción}

$\mathrm{E}$ $\mathrm{n}$ el marco de los enfoques y làs propuestas pedagógicas innovadoras se perfila con bastante fuerza la denominada "pedagogía de proyectos" como una de las opciones que anuncian la recuperación del vínculo escuela-vida en el contexto escolar. En Colombia, el "discurso pedagógico oficial”, desde la misma Ley General de Educación y muchos de los documentos derivados de ésta-los lineamientos curriculares de las distintas áreas, para citar un ejemplo-, e incluso antes, a través de la propuesta que en 1992 se hiciera para el grado cero, ha defendido la importancia de asumir el diseño y la realización de proyectos de aula como materialización de este tipo de "pedagogía", bajo la premisa de que ella permite superar las prácticas tradicionales enmarcadas en la díada transmisionismo-asociacionismo, para darle paso a la construcción de aprendizajes significativos, ligados a la vida de los estudiantes y que parten de los saberes, las necesidades y las inquietudes que éstos se plantean.

Sin embargo, tanto en el ámbito oficial como en los desarrollos teóricos, prácticos e investigativos que sobre el enfoque de proyectos circulan principalmente bajo dos denominaciones, "trabajo por proyectos" o "pedagogía de proyectos", el discurso se caracteriza por la variedad de acepciones tanto del concepto mismo de "proyecto" como de la adjetivación de la que generalmente viene acompañado. En general, se lo inscribe en un marco amplio de relaciones referidas a discursos sobre la investigación en el aula, la integración curricular, la democracia en la escuela, la globalización del aprendizaje y el vínculo escuela-vida. Lo cierto es que la idea de proyecto o, mejor, su uso lexical en el contexto escolar, está presente prácticamente en todo el discurso de la innovación y atraviesa parte de la reforma educativa propuesta en Colombia, al tiempo que es posible identificarla también como parte de las reformas educativas en otros países.

Las reflexiones que se desarrollan a continuación son la síntesis de un trabajo de investigación más amplio, en el que se hace un análisis discursivo de este enfoque de pedagogía de proyectos, tomando como corpus de estudio, primero, unos desarrollos teóricos (principalmente en autores como Jolibert, Not y Hernández); segundo, algunos desarrollos teóricos, prácticos y metodológicos que han tenido lugar específicamente en el área de lenguaje en Colombia, y tercero, una lectura de documentos oficiales del Ministerio de Educación que lo sugieren como propuesta de trabajo pedagógico en el aula. Así mismo, responde a un trabajo de búsqueda y análisis de fuentes primarias en las que se encuentran como génesis del enfoque autores como John Dewey y William Kilpatrich, quienes defienden una concepción pragmática del conocimiento que se denominó en su época project method. ${ }^{2}$

El propósito de este artículo es describir de manera sucinta algunos hallazgos derivados de ese análisis discursivo, en la vía de destacar, en primer lugar, las primeras fuentes y referencias como "método de proyectos"; en segundo lugar, algunos desarrollos teóricos posteriores que ha tenido el enfoque, y, en tercer lugar, la ubicación de algunos de estos desarrollos en el caso del área de lenguaje en Colombia, que aun cuando comparten unos fundamentos en común, se perfilan como perspectivas de trabajo con proyectos en la escuela diferenciadas según sea el énfasis y el sentido pedagógico que comportan o promueven.

Esto último permite identificar la pluralidad de versiones que coexisten bajo una misma denominación en lo que hoy se conoce como "proyecto de aula", y nos permite abrir una discusión en torno a las implicaciones que se derivan no sólo para el área de lenguaje en particular sino para la cultura escolar en general, una vez es asumido, bien sea como estrategia pedagógica de formación, como opción de desarrollo curricular o de integración del mismo, o como modelo pedagógico que promueve la investigación en el marco del desarrollo de un proyecto educativo institucional, en tanto apuesta de imbricación escuela-vida, ligada a la pregunta por la integración de los saberes extraescolares con los saberes escolares.

\footnotetext{
El análisis corresponde a un trabajo de investigación realizado como requisito para optar por el título de $\mathrm{Ma}-$ gíster en Pedagogía. Se inscribe dentro de la línea de investigación denominada "Pedagogía y lenguaje" de la Maestría en Pedagogía de la Universidad Industrial de Santander (Bucaramanga, 2006).
} 


\section{Los orígenes del método de proyectos en la escuela}

Un rastreo de las primeras fuentes y referencias sobre el método de proyectos nos permite ubicar a John Dewey como su inspirador y posteriormente a William H. Kilpatrick en la materialización concreta de la propuesta. Ambos autores, el primero desde un planteamiento filosófico de la educación conocido como "pragmatismo", y el segundo desde esta misma filosofía pero concretada en una perspectiva metodológi$\mathrm{ca}$, se inscriben en una corriente renovadora de la escuela que impugna los modelos transmisionistas, el saber fragmentado que reproducen las disciplinas en la escuela, la pasividad del estudiante y la memorización de contenidos sin aplicación práctica, y propenden por un contenido social de lo que se enseña, por un aprendizaje en, desde y para la vida misma del estudiante. Así, por ejemplo, de un lado, Dewey afirmará, en su idea de la educación como desarrollo natural, que:

Lo que se aprende en la escuela es, en el caso más favorable, sólo una parte pequeña de la educación, una parte relativamente superficial; y sin embargo todo lo que se aprende en la escuela crea distinciones artificiales en la sociedad y aleja a las personas unas de otras. Consiguientemente exageramos el valor de la instrucción escolar, comparada con lo que se gana en el curso ordinario de la vida (1950: 9).

Por otra parte, Kilpatrick, en su teoría pedagógica sobre la redacción del nuevo programa escolar ${ }^{3}$, reflexionando en torno a la relación aprendizaje y vida, propone:

Para comprender cómo el aprender se introduce en la vida tenemos que mirár a la vida y especialmente a la vida fuera de la escuela. Pues a pesar de nuestros prejuicios aca-

3 El programa escolar fue definido en esa época por la $\mathrm{Na}$ tional Society for the Study of Education, de la cual el profesor William H. Kilpatrick junto con otros profesores universitarios hacían parte. En ese momento eran reconocidos como la sociedad pedagógica más importante. démicos, el aprender fuera de la escuela es aún el tipo esencial de aprender, que ha sido siempre y sigue siendo en cantidad e importancia y probablemente en calidad el aprender más importante que realizamos (1944: 39).

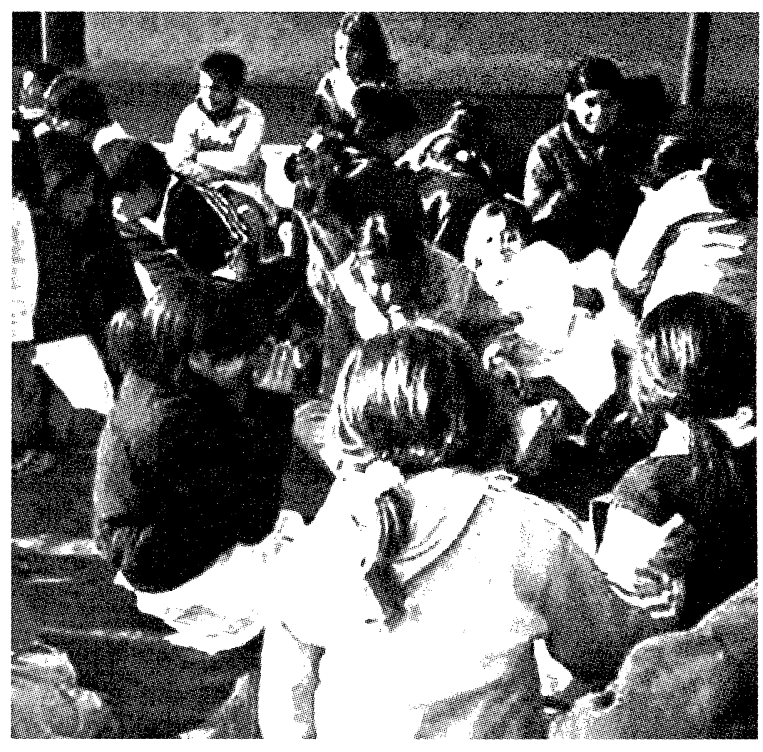

Las coincidencias de los dos autores en la idea de que los impulsos y las necesidades humanas llevadas a la acción son las que conducen a aprender en la vida y para la vida misma son evidentes, y también la idea implícita, que se presenta en consecuencia, de mirar entonces dichos aprendizajes para incorporarlos en la vida escolar. Las asociaciones que establece Kilpatrick entre el aprender en la vida cotidiana y el aprender en la vida escolar lo llevan a suponer que la esencia del aprendizaje es primariamente cambiar el modo de proceder, esto es, un cambio necesario en la conducta y que por ello afecta la vida. Se trata, además, para este autor, no sólo de lo que se "puede hacer" sino de lo que "se quiera hacer" (Kilpatrick, 1944: 43).

Ante la pregunta ¿cómo tiene lugar el aprendizaje?, Kilpatrick pondrá, en primer lugar, la práctica como elemento necesario y, en segundo lugar, la intención del alumno; posteriormente enunciará la asociación en tanto "si dos cosas ocurren juntas con suficiente intensidad, la que se pretende después llamará probablemente a la otra". En consecuencia, el aprender de manera aislada, es decir, alejada de los motivos de los estudiantes, de la experiencia concreta, de la práctica, no es pertinente ni conduce a verdaderos aprendizajes (42-50). 
Dewey, por su lado, va más allá de estos planteamientos, aunque parte de la misma base pragmática del aprendizaje y el conocimiento. Este autor propondrá conceptos como la "acción reflexiva" sobre el ensayo y el error. Más que una experiencia empírica, opta por el conocimiento y el aprendizaje como resultado de la comprobación experimental activa; ello, por cuanto en el fondo de su reflexión considera posible y necesaria una aplicación análoga del método científico en la escuela, partiendo siempre de la experiencia y la acción sobre los objetos del medio circundante. En términos generales, Dewey expone que es la perplejidad que se vive ante una situación nueva y la posibilidad de las anticipaciones que exige -la revisión, la exploración, el análisishasta la formulación de una hipótesis que se proyectará en un plan de acción para experimentar y comprobar directamente, lo que da lugar al pensamiento y que posteriormente acaba siempre en conocimiento (Dewey, 196̈7: 153-166).

Sin embargo, en una revisión actual del método de proyectos, éste es seriamente cuestionado no sólo como método en sí mismo sino como aplicación de las ideas del propio Dewey. Algunas de esas inconsistencias son señaladas por Torres (1994), quien en su perspectiva de análisis del currículo lo clasifica como formas tradicionales de integración y señala que la intención de Kilpatrick es convertir el currículo de cada curso en un conjunto de proyectos que suponen cuatro pasos: decidir el propósito del proyecto, realizar un plan de trabajo para su resolución, ejecutar el plan diseñado y juzgar el trabajo realizado. La educación tradicional, según este gestor del método, se equipara aquí a una baraja de conocimientos memorísticos pero sin ningún grado de utilidad: "en aquella escuela, en primer lugar se daba a los alumnos los conceptos científicos, leyes, definiciones, métodos de investigación, etc., en lugar de ser inducidos del resultado de su propia acción en equipo". Así pues, según Torres, el punto de arranque dèl método es fruto de la siguiente filosofía: “¿por qué no hacer dentro del aula lo que se hace continuamente en la calle, en el verdadero medio natural?" (1994: 202).

El método de proyectos se presenta entonces como un conjunto de oposiciones subsecuentes: educación tradicional/educación nueva, memori- zación/acción y saber escolar/saber cotidiano, en el que es este último el que prevalece, en tanto:

El método de proyectos se desarrolla, en consecuencia, con la finalidad de dar solución a los problemas que chicos y chicas se plantean en su vida cotidiana y que necesitan resolver, tales como, por ejemplo, levantar una choza, preparar una fiesta local, construir una pequeña granja, ayudar a un animal herido, etc. [...] El saber con el que se trata en las aulas pasa a ser el mismo que se utiliza en la vida real, sólo que allí cobra importancia especial la reflexión y la ordenación de esa realidad para hacerla asequible al conjunto de estudiantes (Torres, 1994: 200).

Las objeciones que encuentra Torres al respecto van, en primer lugar, en la línea de coincidencia histórica con las ideas de aprendizaje de Thorndike, según las cuales éste se produce por conexiones estímulo-respuesta y por asociación, tal y como se pretende con el método de proyectos que lleva a reducir los propósitos educativos a la construcción de respuestas adecuadas al medio: este precisamente es, según el mismo autor, el punto de contradicción con el referente deweyano. En segundo lugar, la crítica va encaminada al paidocentrismo en el que se cae, dado que la responsabilidad última en la decisión sobre el aprendizaje corresponde a los estudiantes. Éste será el principal problema de dicha modalidad de integración curricular, derivado de sus presuposiciones de base, en las que se deja de lado la participación del profesorado en la planificación y en la evaluación pertinente de los conocimientos, destrezas, habilidades y valores que los alumnos y alumnas necesitan construir; de este modo, en muchas de las propuestas sugeridas por los estudiantes se exalta la diversión y el placer, pero resultan triviales desde el punto de vista educativo (Torres, 1994: 203).

Visto de esta manera, aún cuando el método de proyectos y las reflexiones de donde se deriva surgen en contraposición con la educación tradicional, su génesis se circunscribe, de un lado, a la concepción asociacionista del aprendizaje y, de otro, al pragmatismo e instrumentalismo de la educación, que tiene como corolario la idea de que el conocimiento genuino en la escuela es sólo aquel que se torna útil para las contingencias de 
la vida. Por ello, en la realización práctica de la propuesta, los dos autores insistirán en partir de las actividades de la vida cotidiana y de las experiencias concretas; insistirán también en la necesidad de crear microcomunidades que reproduzcan los valores de la comunidad para efectos de ir construyendo "principios democráticos" y en suprimir el dualismo en los planes de estudio que suponen una separación del individuo en la escuela y en la vida (Dewey, 1967: 206).

\section{Desarrollos teóricos posteriores del enfoque de proyectos}

El enfoque de proyectos, ya sea en su versión original como "método" o en sus denominaciones más recientes en las que circula como "trabajo por proyectos" o "pedagogía de proyectos", sigue siendo un referente del discurso de la innovación educativa de los últimos años, en oposición a la educación tradicional. Sin embargo, aun cuando los desarrollos teóricos, prácticos e investigativos que han difundido el discurso de los proyectos en la escuela tienen sus antecedentes en la propuesta originaria del método de proyectos, toman orientaciones conceptuales y pedagógicas diferenciadas y tienden puentes de relación distintos, como se observará adelante.

Así, por ejemplo, para Hernández y Ventura (1992) la asunción de los proyectos de trabajo en la escuela comporta una apuesta fundada en la concepción de aprendizaje significativo y del procesamiento de información para lograr la globalización de los aprendizajes, esto es, la construcción de un

Para Jolibert (1995) la pedagogía de proyectos constituye una estrategia formativa que permite romper con el modelo de la escuela tradicional y con los roles de maestros y alumnos, e instaurar una apuesta democrática y un proceso pedagógico en el que todos participan proceso de integración de saberes que hace el estudiante como parte de la correlación y reelaboración de su conocimiento previo, frente a la potencialidad de relación que pueda darse con los materiales y los nuevos contenidos ofrecidos. Sus premisas pasan por la comprensión de la perspectiva ausbeliana del "aprendizaje significativo" y sus fundamentos cognitivos, y la integración de esta concepción en una actitud flexible frente al currículo, por cuanto la globalización del conocimiento "requiere que el tema o el problema abordado en la clase sea el factor en el que confluyan los conocimientos que respondan a las necesidades de relación que el alumnado puede establecer y el docente ha de interpretar" (Hernándeż y Ventura, 1992: 55).

De esta manera, la organización del currículo en proyectos de trabajo responde a una visión desde la estructura cognoscitiva de los estudiantes; en esa medida no está referido a las disciplinas escolares, ni a la ordenación y secuenciación de los contenidos en sí mismos, aunque de hecho éstos se contemplan de acuerdo a lo que podría esperarse en los alumnos, según los niveles y ciclos en los que se encuentren. Los proyectos buscan aquí, fundamentalmente, favorecer la creación de estrategias de organización de los conocimientos escolares en función del tratamiento de la información y la relación entre los diferentes contenidos en torno a problemas e hipótesis. Las actividades y estrategias procedimentales desarrolladas a través de los proyectos de trabajo tienen como base una concepción de proceso más interna y psicológica, en función de un aprendizaje que adquiera valor relacional y comprensivo, posibilitando de esta manera el desarrollo del principio de la globalización como estructura psicológica del aprendizaje (Hernández y Ventura, 1992).

Entre tanto, para Jolibert (1995) la pedagogía de proyectos constituye una estrategia formativa que permite romper con el modelo de la escuela tradicional y con los roles de maestros y alumnos, e instaurar una apuesta democrática y un proceso pedagógico en el que todos participan desde la misma planificación hasta la ejecución y evaluación del proyecto, como una manera también de lograr aprendizajes significativos que, además, son susceptibles de ser desarrollados al interior de un área específica -Lengua Materna- con una didáctica consecuente con este enfoque pedagógico. 
fasis exclusivo en el aprendizaje contextualizado ${ }^{6}$; se trata más bien de una concepción que acepta el marco de los contenidos culturales propios de la escuela, pero que pretende resignificar la relación pedagógica en tanto relación comunicativa e intersubjetiva.

De lo expuesto en las tres perspectivas teóricas, se colige entonces que "lo pedagógico" de los proyectos es su potencialidad para la concreción del aprendizaje significativo del alumno, a quien se le otorga otro lugar en su participación y en la toma en cuenta de sus intereses, necesidades, motivaciones y conocimientos previos. Las concepciones que se juegan en la relación didáctica maestroalumno-objeto de conocimiento provienen todas de un fundamento psicológico $\mathrm{y}$, especialmente, de un enfoque cognitivo del aprendizaje, aunque en una pluralidad de versiones del mismo (Piaget, Vygostki, Ausubel y Coll, entre otros). ${ }^{7}$
Así, aunque los proyectos remiten a lugares distintos al ser enunciados como concepción educativa (Hernández y Ventura), como pedagogía (Jolibert) o como concreción de "una educación en segunda persona" o enseñanza dialogante (Not), en general, su sentido pedagógico se fundamenta en una "psicología del aprendizaje" y se concreta en propuestas de orden metodológico que, no obstante, resignifican las relaciones y las posiciones en el ámbito escolar del maestro y del alumno, las concepciones de enseñanza y aprendizaje, las miradas sobre los objetos de conocimiento, las disciplinas escolares, el currículo y la evaluación en general, pero que operan en conjunto como una recontextualización del discurso constructivista en la educación. La siguiente tabla ilustra a manera de síntesis lo expuesto:

\begin{tabular}{|c|l|l|l|}
\hline Denominación y Autores & \multicolumn{1}{|c|}{ Adscripción teórica } & \multicolumn{1}{c|}{ Enfoque } & \multicolumn{1}{c|}{$\begin{array}{c}\text { Tipo de proyectos e interés } \\
\text { cognoscitivo }\end{array}$} \\
\hline $\begin{array}{c}\text { Trabajo por proyectos } \\
\text { Fernández y Ventura }\end{array}$ & $\begin{array}{l}\text { Movimiento progresista en } \\
\text { educación (Dewey). } \\
\text { Enfoques cognitivos del } \\
\text { aprendizaje. }\end{array}$ & $\begin{array}{l}\text { Globalización del } \\
\text { aprendizaje. }\end{array}$ & $\begin{array}{l}\text { Proyectos para el acceso y } \\
\text { manejo de la información y el } \\
\text { aprendizaje significativo. } \\
\text { (Prima el interés teórico y } \\
\text { técnico.) }\end{array}$ \\
\hline $\begin{array}{c}\text { Pedagogía de proyectos } \\
\text { Jossette Jolibert }\end{array}$ & $\begin{array}{l}\text { Pedagogías activas del siglo } \\
\text { xx (Dewey, Freinet). } \\
\text { Constructivismo en } \\
\text { perspectiva psico y } \\
\text { sociocultural. }\end{array}$ & $\begin{array}{l}\text { Estrategia de formación y } \\
\text { desarrollo curricular de un } \\
\text { área específica: la lengua } \\
\text { materna. }\end{array}$ & $\begin{array}{l}\text { Proyectos para el aprendizaje } \\
\text { de la lengua materna en } \\
\text { contextos significativos. } \\
\text { (Prima el interés práctico y } \\
\text { emancipatorio.) }\end{array}$ \\
\hline $\begin{array}{c}\text { Pedagogía del proyecto } \\
\text { Louis Not }\end{array}$ & $\begin{array}{l}\text { Constructivismo } \\
\text { psicogenético } \\
\text { de Jean Piaget. }\end{array}$ & $\begin{array}{l}\text { Relación pedagógica- } \\
\text { comunicativa en } \\
\text { segunda persona: } \\
\text { interestructuración del } \\
\text { conocimiento. }\end{array}$ & $\begin{array}{l}\text { Proyectos de conocimiento } \\
\text { propiamente escolar. } \\
\text { (Prima el interés teórico, } \\
\text { técnico, práctico y } \\
\text { emancipatorio.) }\end{array}$ \\
\hline
\end{tabular}

6 De hecho, el autor ha posicionado en otro trabajo suyo (Las pedagogias del conocimiento, 1979) unos puntos críticos frente a la "escuela activa" en general y que ubica en la categoría autoestructurante del conocimiento.

7 Si bien es en Louis Not en donde se perfila una adscripción teórica específica, la proveniente de la psicología genética de Jean Piaget conocida como constructivismo psicogenético, Hernández y Ventura o Jolibert refieren de manera más general sus adscripciones, los primeros en una asunción de un enfoque plural de teorías cognitivas sobre el aprendizaje (Hernández y Ventura, 1996: 52-55 y Hernández, 2002) y el segundo en una integración de teorías psico y sociocontructivistas (Jolibert, 1999).
8 La distinción entre los tipos de intereses está categorizada en términos habermasianos, específicamente en sus elaboraciones en torno a la relación "conocimiento e interés". Usamos estas categorías con el propósito de mostrar unos énfasis de los proyectos en términos de dicha relación, entendiendo el interés teórico y técnico como la intermediación directa con el mundo físico, sensible y observable; el práctico como praxis social en el sentido de una interacción personal y social dentro del contexto histórico que se vive, $y$, finalmente, el emancipatorio como equivalente a la liberación de las dependencias de los sistemas hegemónicos, develando teórica y comprensivamente el factor de poder que estructura toda interacción social (Habermas, 1997). 


\section{Desarrollos teórico-prácticos del enfoque de proyectos: el caso del área de Lenguaje}

Si bien son numerosos los desarrollos teóricos y prácticos que sobre el trabajo por proyectos o la pedagogía de proyectos -sigue teniendo en el discurso ambas denominaciones- se han producido en Colombia en los últimos años, 'es importante señalar cómo particularmente en el área de Lenguaje, y específicamente en la didáctica de la lengua y la literatura, el enfoque ha sido promovido y desarrollado con mayor fuerza. ${ }^{9}$ Grosso modo, estas son algunas de las variantes identificadas a partir de los discursos que convocan el enfoque de proyectos en este campo disciplinar (ver cuadro síntesis):

\subsection{Trabajo por proyectos y enseñanza del lenguaje escrito}

Esta relación se halla inscrita en la perspectiva de comprender, según Rincón, que "la lengua no se restringe a un área escolar sino que está presente en el desarrollo del currículo por lo menos de tres maneras: como sistema de comunicación de la clase y la escuela, como medio de aprendizaje, como objeto de conocimiento" (Rincón, s.f.: 41). ${ }^{10}$ Para la autora, la lógica escolar que aísla las

9 La fuente que ha servido de base para alcanzar ciertos niveles de trabajo pedagógico a partir de proyectos en el área de lenguaje probablemente la constituye la obra de Josette Jolibert, quien iniciara, primero en Francia (con el grupo de docentes Ecouen), la apuesta por una educación más activa y constructiva y luego, con el respaldo de la UnESCO, se propusiera la fundación de una red latinoamericana de maestros para promover la pedagogía de proyectos en el área de lenguaje. Actualmente Colombia hace parte de esta red y en su territorio articula once nodos que tejen a su vez la red a nivel nacional (conformada en 1994), cuyos propósitos de cualificación y transformación de las prácticas docentes en la didáctica del lenguaje y la literatura se materializan en el marco del desarrollo y sistematización de proyectos (en: http:// www.mineducacion.gov.co/1621/article-122254.html)

${ }^{10}$ Estas reflexiones son retomadas de una publicación de la Escuela de Ciencias de Lenguaje (Universidad del Valle), en el contexto del Programa de Mejoramiento Docente en Lengua Materna (financiado por el Fondo Men-Icetex), y que se titula: "El trabajo por proyectos y áreas de enseñanza ignora muchas veces también el papel que cumple la lengua escrita como mediadora de los procesos de aprendizaje de todas las disciplinas. En ese sentido, la concepción del trabajo por proyectos se asume como "una opción pedagógica que al pretender un alto grado de integración ofrece enormes posibilidades al desarrollo de las competencias cognitiva y comunicativa de los maestros y alumnos" (9). La integración se puede leer aquí en dos sentidos posibles: de un lado, como integración (globalización) de conocimientos provenientes de las diversas disciplinas escolares y, de otro, como integración de los intereses de los niños y de sus propuestas en su relación con los saberes escolares.

Así mismo, Rincón señala que introducir el trabajo por proyectos en la escuela "no es sólo un asunto técnico", pues implica cambios en la forma como se establecen las relaciones de poder, de saber y de afectividad entre autoridades educativas, maestros, alumnos, padres de familia y comunidad educativa en general. Exige también ambientes de trabajo cooperativo, solidario $y$ tolerante en los que permanentemente se practique la democracia, la valoración de la diferencia y la responsabilidad (3).

En esta perspectiva, el trabajo por proyectos es una manera de empezar a cambiar la concepción de currículo de un plan de estudios dividido en asignaturas o materias avanzando hacia un modelo de integración curricular (20-21). En su relación específica con el plan de estudios se propone una tipología de proyectos, según sea su naturaleza interárea, es decir, abordando contenidos de diferentes áreas o de naturaleza intraárea, es decir, con contenidos específicos del área de lenguaje a propósito de un tema-problema que ha sido planteado. De cualquier modo, sean de naturaleza intra o interárea, las situaciones de lectura y escritura que convocan la realización de un proyecto se constituyen en actividades permanentes del mismo, por lo cual se espera que "al mismo tiempo que se va aprendiendo (implícita o explícitamente) sobre las estructuras, funcionamiento y funciones de diversos tipos de textos se vayan apropiando las condi-

la enseñanza y el aprendizaje del lenguaje escrito en la educación primaria" (s.f.). 
ciones pragmáticas necesarias para su adecuación a una situación comunicativa determinada" (40). ${ }^{11}$

En la concreción metodológica de la propuesta la autora plantea que aunque existen diversas formas de realizar un proyecto, es necesario tener en cuenta unas fases o momentos para su desarrollo. Básicamente destaca la "planificación conjunta", que incluye la toma de decisiones sobre la problemática a abordar, los objetivos que orientarán el proyecto y las actividades o medios para realizarlo. Ésta se lleva a cabo en conjunto con los niños y deja de ser una práctica exclusiva del maestro. En ese sentido, se sugiere una serie de preguntas que sirven como organizadoras de la actividad: "qué vamos a hacer o aprender? (tema), qué queremos saber sobre esto? (subtemas), por qué (objetivos e hipótesis), cómo?(actividades), cuándo?(cronograma), dónde? (espacios), con quiénes? (invitados o colaboradores), con qué (recursos), cómo vamos a denominar nuestro proyecto? [sic]” (Rincón: 28).

La segunda fase es la "ejecución", que consiste en el desarrollo del plan previsto, y la tercera fase es la "evaluación conjunta", que se vive, según esta perspectiva, de manera permanente por cuanto se está evaluando el cumplimiento de la planificación propuesta. Al igual que las demás fases ésta última es "responsabilidad de maestros y alumnos" y debe tener en cuenta: "los procedimientos que se pusieron en práctica, las actitudes $\mathrm{y}$ los valores que se fomentaron, las interacciones que se posibilitaron, el logro de los objetivos y las competencias construidas" (37).

\subsection{Entre el proyecto pedagógico y el proyecto de aula}

Este enfoque, que integra tanto el proyecto pedagógico del maestro como el proyecto de aula entre alumnos y docentes, se sustenta en una con-

${ }^{11}$ En esta perspectiva se inscribió el "Programa de mejoramiento docente en lengua materna" (Univalle-Men) y se realizaron, sistematizaron y publicaron experiencias de proyectos de aula a través de la serie "Maestros escribiendo". cepción de la pedagogía de proyectos como "estrategia de trabajo que facilita la inserción de la escuela en la vida, permitiendo el desarrollo personal, la adquisición de saberes y la formación de individuos partícipes y autónomos" (Rodríguez, 2001: 17).$^{12}$ Así mismo, se postula como una opción de cambio en la cultura escolar y como concepción teórico-metodológica flexible y práctica que permite alcanzar las metas institucionales $y$, al mismo tiempo, dar respuesta a las necesidades individuales (26). El enfoque ubica esta perspectiva pedagógica en una concepción general de la enseñanza y el aprendizaje que concilia "los intereses y necesidades subjetivas de los educandos y los propósitos objetivos de la institución" (26).

Tomando como fundamento experiencias históricas como el Plan Dalton (1911) y el método Winnetka, así como a una amplia gama de autores como Dewey, Freinet, Jolibert, Le Grain, Vassileff y Touchon, entre otros, se reivindica en este discurso la pedagogía de proyectos por cuanto favorece -según Rodríguez-la no atomización de los aprendizajes y la apropiación significativa de los saberes. No obstante, también se reconocen los cuestionamientos que se le han hecho y que se identifican con aspectos referidos a la poca sistematización en los aprendizajes, a la falta de rigor en el tratamiento de los contenidos, a la desviación del papel del docente, al reduccionismo pragmático que puede conducir a una representación falsa del desarrollo cognoscitivo al equipararlo con el activismo pedagógico. Deficiencias que según la autora pueden ser superadas con la investigación didáctica, pues finalmente la propuesta no

\footnotetext{
${ }^{12}$ Estas reflexiones se realizan en el marco de un programa formativo de la Especialización en Lenguaje y Pedagogía de Proyectos que ofrece la Universidad Distrital Francisco José de Caldas. Del programa formativo se deriva la realización y sistematización de proyectos que se publican en la serie Cuadernos de trabajo de la Especialización en la que además se presentan artículos y experiencias referidos a esta temática en su especificidad desde el área de Lenguaje. Algunos de estos documentos constituyen el referente de este apartado.
} 
está centrada solamente en el propósito de "conducir a los alumnos hacia un cierto nivel de conocimientos y competencias, sino el de integrar esos saberes en la lógica de su proyecto" (2001: 27).

En la concepción interaccionista que se defiende como sustento de este tipo de pedagogía está el concepto vigotskiano de "zona de desarrollo próximo" y de "ámbito de actividad" de Gallimore y Tharp. También se sustenta, con Jolibert, una concepción de la didáctica en este marco-de pedagogía de proyectos como "campo específico, teórico-práctico, que se refiere a la construcción y manejo de los aprendizajes en el aula”. En esta perspectiva, el rol del docente cumple unas funciones específicas pero se reitera como horizonte general su papel de "formador"; así, la experiencia del trabajo por proyectos se relaciona con una necesidad sentida de cualificación de los docentes "para generar los conocimientos específicos en su labor didáctica y no continuar siendo aplicadores de teorías especializadas al margen de la actividad escolar".

Desde este enfoque, el proyecto de aula nace en primer lugar de un proyecto pedagógico liderado por un maestro o un colectivo docente en el área de Lenguaje, guiado por una pregunta de investigación que plantea una situación problemática, en la perspectiva de conocer y comprender críticamente el contexto escolar y de cons- truir conocimiento pedagógico que reoriente la práctica y reivindique el compromiso intelectual de los maestros. La perspectiva de investigación que se asume aquí es la de investigación-acción participativa con elementos de la etnografía, en la que instrumentos como la observación, el diario de campo, las entrevistas, etc., toman vigencia. No obstante, todos los proyectos delimitan unas posibles fases o momentos en los que se puede leer el origen del proyecto, la etapa de negociación y planificación con los niños, la búsqueda de información y desarrollo del trabajo, y la socialización del proyecto a través de la presentación de un producto final (2001: 45-160).

\subsection{Proyectos de aula e investigación cooperativa escuela-universidad}

Esta relación de los proyectos de aula con la investigación cooperativa entre docentes universitarios y docentes de Educación Básica y Media surge en el marco del Programa de Fortalecimiento de la Capacidad Científica en la Educación Básica y Media (RED), definido como una propuesta de investigación interdisciplinaria en torno a la escuela y realizado en forma cooperativa por profesores de la Universidad Nacional de Colombia y de la Educación Básica y Media de diferentes regiones del país.

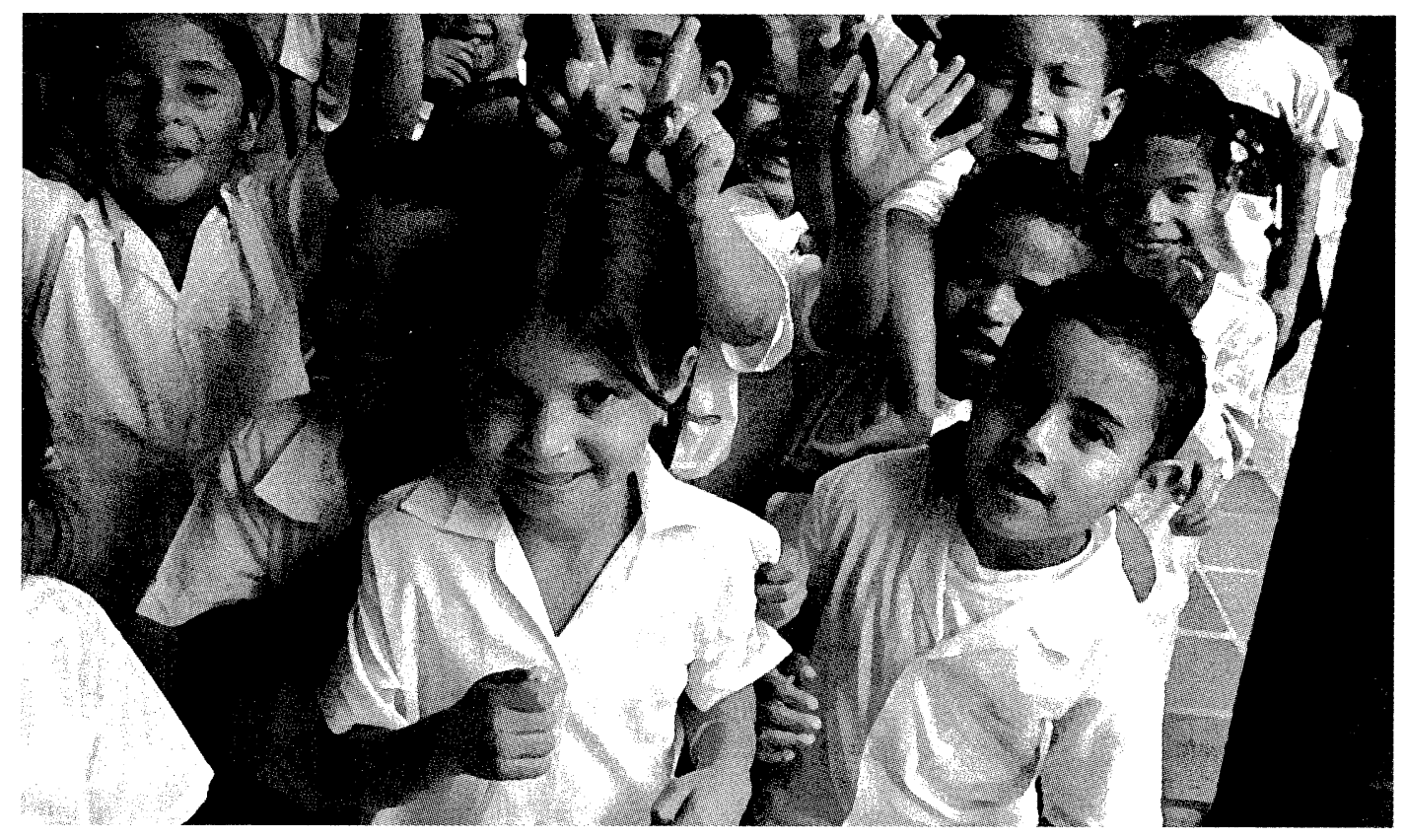


Los proyectos de investigación del Programa RED tienen tres dimensiones: los procesos pedagógicos, la vida cotidiana de la escuela y la dimensión institucional. La construcción del objeto de investigación supone un proceso de interlocución y debate entre los principales actores, que pasa por la mirada en torno a los procesos pedagógicos, pero también implica una visión general de la escuela en sus ámbitos de gestión, dirección y organización; es por ello que el ejercicio de objetivar el quehacer pedagógico para problematizarlo y decantar desde allí unos objetos específicos no es posible -según esta perspectiva- desde la mirada unidireccional de los profesores universitarios, sino que exige fundamentalmente la participación tanto de los profesores como de los directivos (Acevedo, et al., 1998: 23-24).

Vistas desde este escenario, la imbricación de las tres dimensiones expuestas y la integración de los diferentes proyectos (de investigación, educativo institucional y de aula) bajo una perspectiva en común se constituyen en una orientación del programa. Sin embargo, no se describe éste como un proceso lineal y exento de dificultades, sino que, por el contrario, se reconoce que el proyecto de investigación es un lugar donde confluyen preocupaciones y demandas de los diferentes actores y contextos y por ello es también un lugar de tensiones no resueltas "entre intereses personales (tanto del profesor del colegio como de la universidad), las prioridades institucionales (provenientes tanto de los PEI como de los planteamientos de RED) y las exigencias del medio y el contexto (comunidad educativa, exigencias de las autoridades)" (29). La investigación se construye de este modo en un ambiente de tensiones, en una serie de desplazamientos continuos y en un permanente contraste entre la teoría y la práctica, entre la práctica y la teoría.

En la reflexión específica para el área de Lenguaje y Literatura se sustenta una concepción de proyecto con un claro horizonte investigativo. Para Jurado Valencia (1999) hay una diferencia sustancial entre un programa y un proyecto de aula o de área, y entre éstos y el proyecto educativo institucional:

Mientras que el programa surge de una planeación etnocentrista, emanada desde una instancia superior, como lo es el Ministerio, el proyecto tiene que surgir de la iniciativa del profesor, de su discusión entre pares y de su inserción en el proyecto educativo de la institución... Mientras que el programa connota la organización de contenidos y objetivos, en listados, que tienen que cumplirse, de acuerdo con unos ritmos y tiempos fijos, para una población homogénea, el proyecto al contrario, connota incertidumbre, horizonte investigativo y seguimiento pausado para una población heterogénea. Si un determinado programa significa planeación para ejecutar, un determinado proyecto significa poner a prueba conjeturas, dar cuenta de lo que va ocurriendo en el proceso, intentar un cambio (91-92).

En esta perspectiva, el proyecto reclama una actitud investigativa por parte del maestro y propicia condiciones para repensar los campos específicos de formación en relación con las comunidades, según el tipo de educación que se demanda y se prioriza en la perspectiva de un proyecto social. En el contexto escolar propiamente dado y en la relación de los proyectos de aula con campos específicos de estudio, esta perspectiva sugiere la necesidad de una sólida formación disciplinar para, desde allí, primero, posibilitar los encuentros y los diálogos con los maestros de una misma área, que permitan vincular los proyectos de aula con un enfoque y una propuesta más general (el proyecto de área) y, segundo, posibilitar los diálogos con maestros de otras áreas, abonando el terreno para lo que puede constituirse posteriormente en una mirada interdisciplinaria de los proyectos.

\section{A manera de conclusiones}

De los diferentes planteamientos que son el sustrato del discurso de los proyectos en la escuela se puede señalar, en primer lugar, que el enfoque se sitúa en un marco más amplio de la educación que se deriva históricamente del Movimiento de Educación Nueva (pedagogía activa), de corte progresista y de una filosofía pragmática del conocimiento y, en segundo lugar, que en sus desarrollos más recientes integra teorías provenientes de la corriente constructivista, de 
carácter cognitivo y sociocultural; que centra su mirada en el alumno y en sus procesos de aprendizaje. Así, dos tipos de fundamentos integran entonces el enfoque de proyectos en la escuela: uno, derivado de unos "supuestos filosóficos" en la educación: el pragmatismo; otro, proveniente de unos "supuestos psicológicos" en torno al aprendizaje: el constructivismo.

La concepción de proyecto, sea en su versión original como método o en sus posteriores desarrollos, integra en su discurso ambos supuestos y mantiene en sus enunciados un presupuesto básico: la necesidad de vincular la escuela con la vida. Para tales efectos, se anuncia la necesidad de integrar en la cultura escolar los saberes experienciales o del "mundo de la vida" de los estudiantes. El enfoque de proyectos intenta desdibujar así las fronteras entre maestros y estudiantes, entre saberes escolares y extraescolares, entre saberes para la escuela y saberes para la vida, en un marco que se presume democrático. En esa medida, se le puede identificar con la clasificación propuesta por Basil Bernstein (1985) como una "pedagogía invisible". ${ }^{3}$

Frente al supuesto pragmático del conocimiento se llama la atención, en tanto puede conducir a un desconocimiento de la tradición o de la propia cultura escolar. Si en éste prima la idea de que el único conocimiento válido y genuino en la escuela es aquel que se torna útil para las contingencias de la vida, el resultado es que un alto porcentaje de los contenidos básicos de la escuela y que proveen una cultura general, un acercamiento al mundo social, natural y físico, un desarrollo del pensamiento y del lenguaje críticos y reflexivos, tendría entonces que ser desechado. Así, las posibilidades de democratización del sistema edu-

\footnotetext{
${ }^{13}$ Este marco se hace desde la teoría sociológica de la transmisión cultural de Basil Bernstein. Este autor, interesado en mostrar las dinámicas de poder y de control que subyacen a las modalidades de transmisión cultural, asumidas como formas sociales y como contenidos específicos, sostiene que la oposición entre las pedagogías conservadoras o tradicionales frente a las pedagogías progresistas o centradas en el niño, que denomina Pedagogías Visibles (PVs) y Pedagogías Invisibles (PIs) correspondientemente, se mueven siempre en una lógica interna de reglas que definen y distinguen un tipo de relación pedagógica y por tanto un tipo de transmisión cultural sobre otra.
}

cativo se tornan por esta vía cada vez más utópicas. ${ }^{14}$ Así mismo, frente al constructivismo como un núcleo común que sustenta las bases psicológicas del aprendizaje en la escuela y que se integra como el fundamento de los enfoques más recientes en torno a los proyectos, habría que sostener que no puede reducirse a una generalidad, pues cada una de las teorías remite a versiones distintas de esta corriente epistemológica. ${ }^{15}$

Interpelar estos fundamentos y supuestos es necesario para no caer en una asunción ligera de una educación por proyectos, pues cabe el riesgo de que tal enfoque conduzca a un extremo: hacer cosas sin prospección cognitiva. Quizá esto sucede en algunas de las experiencias que se tienen sobre proyectos de aula, en las que no hay un control sobre los conceptos fundamentales que en el plano cognitivo han de ponerse en juego. De esta manera, la pedagogía de proyectos puede caer en el activismo y en el estereotipo, si no propende por ayudar a construir los conocimientos universales.

\section{El enfoque de proyectos intenta desdibujar así las fronteras entre maestros y estudiantes, entre saberes escolares y extraescolares, entre saberes para la escuela y saberes para la vida, en un marco que se
presume democrático.}

${ }^{14} \mathrm{El}$ prinçipio deweyano de "aprender haciendo" a partir de la experiencia cotidiana y directa, antes que aprender el conjunto de materias propias de la cultura escolar, deja a los grupos privilegiados con más posibilidades que a los grupos que provienen de las clases más deprimidas, precisamente por el tipo de experiencias a las que han tenido acceso.

${ }^{15}$ Castorina (1987) señala, por ejemplo, que términos como aprendizaje, actividad, esquema e idea previa, entre otros, remiten a una trama conceptual distinta en cada teoría o autor y es allí donde adquieren su sentido; la generalización de los mismos puede, según el autor, "ocultar diferencias conceptuales sustantivas entre las teorías agrupadas de ese modo" (Castorina, 1998: 26). 
Estamos entonces frente a un dilema pedagógico muy recurrente y del cual se derivan muchas de las tensiones de la vida escolar: entre la individualidad y la diversidad cultural, por un lado, y la homogeneidad y la cultura universal, por el otro. La pregunta que queda es cómo, desde una perspectiva pedagógica de trabajo con proyectos en la escuela, se logra una regulación común sobre la selección cultural que se hace, al tiempo que se propicia una relación genuina entre el sujeto, la escuela y la comunidad.

Esto último, teniendo en cuenta que desde algunas de sus perspectivas la pedagogía de proyectos puede operar como una Pedagogía Invisible, en la medida en que, tanto en su discurso instruccional (secuencia, ritmo y criterio) como en su discurso regulativo (relación maestro-alumno), flexibiliza y negocia las reglas de selección y secuenciación de los contenidos, así como también promueve una relación horizontal en la que el maestro apàrece como un orientador y facilitador de procesos que aprende en conjunto con sus estudiantes, definiendo en últimas y por consenso el texto legitimo a aprender y enseñar, lo cual implica cambios no sólo en la selección de los contenidos curriculares, sino en la organización de lo que se adquiere y, por tanto, en las representaciones que se derivan de ello.

De igual forma, sostenido este enfoque por teorías desarrollistas, en las que el niño es partícipe de su propio aprendizaje y en las que se tiende a reemplazar "la dominación por la facilitación, la imposición por la acomodación" (Berstein, 1985: 44), el producto de la formación no se regiría entonces por un patrón estándar común y externo. Esta última acotación nos lleva a la dicotomía que se produce en el seno del discurso pedagógico oficial en Colombia, que por un lado legitima la opción del trabajo con proyectos -sin asumir las implicaciones y las condiciones de posibilidad que ello amerita-, al tiempo que promueve una estandarización oficial de los contenidos curriculares y una legitimación de los mismos por la vía de la evaluación de competencias. Nos parece que estos cuestionamientos abren un campo de discusión no sólo importante, sino muy pertinente en la coyuntura actual de lo que es nuestro sistema educativo.
Por otro lado, este recorrido discursivo por la pedagogía de proyectos situado ya en sus desarrollos teóricos y prácticos en el caso del área de Lenguaje, permite también caracterizar el enfoque y sus posibles variantes. Lo que aparece como constante en los discursos que lo fundamentan es, precisamente, una apropiación diversa del mismo y unos desarrollos que se corresponden con versiones distintas de lo que se concibe y se materializa finalmente como "proyecto de aula" en la escuela.

En ese sentido se tiene, primero, en la perspectiva del trabajo por proyectos y la enseñanza del lenguaje escrito, un énfasis ligado a la integración curricular, al tiempo que los procesos lectoescritores retoman un sentido distinto en la vía de resolver el tema-problema del proyecto de aula. Segundo, desde su asunción como pedagogía de proyectos en el marco de la investigación-acción en el aula, se presenta como una resignificación del papel intelectual del maestro $y$ del diseño de sus proyectos de aula como parte constitutiva de sus proyectos pedagógicos. Por último, el proyecto de aula remite directamente a un proyecto de investigación del maestro, derivado al tiempo de un proceso de formación disciplinar e investigativa, que se articula con una política que intenta ligar la investigación de la universidad con la investigación en la escuela.

En síntesis, en las perspectivas descritas los proyectos aparecen como paradigma de la innovación en la escuela y como oposición a la pedagogía tradicional, y están motivados de cierta manera por la necesidad de construir otras opciones y estrategias pedagógicas que partan, así mismo, del reconocimiento de los contextos socioculturales de los estudiantes. En ese sentido, el discurso sobre los proyectos se perfila también aquí como una aspiración a superar esta ruptura u oposición entre la experiencia cotidiana y la experiencia de la escolarización, y opera como una suerte de recontextualización de los orígenes $y$ fundamentos de este enfoque.

Sin embargo, el hecho relevante es que estos discursos surgen a partir de experiencias de formación docente, en el contexto de programas de mejoramiento o de especialización en un campo de saber específico: el lenguaje. De esta manera, los maestros tienen la posibilidad de asu- 
mir el enfoque comprensivamente -según sea la perspectiva pedagógica desde donde se enunciaen tanto ha mediado un proceso formativo que pasa por la apropiación teórica hasta su desarrollo práctico en las instituciones escolares a través del diseño, realización, sistematización y publicación de sus proyectos de aula. Se trata de procesos de formación a largo plazo y la diferencia aquí es sustancial, por cuanto los desarrollos más elaborados que se encuentran son precisamente producto de este tipo de procesos formativos.

De ello se deduce que no se puede esperar la incorporación de una pedagogía de proyectos en la escuela por parte de los docentes como una exigencia de cumplimiento de la norma. Su asunción implica la revisión de los procesos de formación tanto inicial como continua, en los que no se trata de "capacitar" a los maestros en "proyectos de aula" a través de talleres aislados o con un énfasis en lo metodológico. Esta es quizá también la nota predominànte una vez un discurso se ha posicionado y legitimado oficialmente, pues aparecen "expertos" enseñándole a los maestros cómo dejar de ser "tradicionales" para convertirse de un día para otro en "innovadores". Se trata entonces de profundizar en un enfoque pedagógico que, como se demostró, toca diversos aspectos de la escuela e incluso redimensiona la cultura escolar y la manera como se agencia con los estudiantes, y redimensiona también los procesos de enseñanza y de aprendizaje, los objetos de conocimiento y las interacciones comunicativas en el aula. Finalmente, si aludiendo a sus bondades lo que se intenta es promover esta pedagogía, es ineludible entonces la discusión amplia con los maestros sobre sus orígenes, fundamentos y supuestos, sobre sus implicaciones pedagógicas $y$, por ende, sobre sus posibilidades en nuestro sistema escolar. Ó

\section{Bibliografía}

Acevedo, et al. (1998), "La investigación en la escuela", en Programa de fortalecimiento de la capacidad cientifica en la educación básica y media, Bogotá, Universidad Nacional de Colombia, programa RED.
Agudo, S. et al. (2000), Interacción y competencia comunicativa. Experiencias sobre lectura y escritura en la escuela, Bogotá, Universidad Nacional de Colombia, Programa Red.

Beltrán, F. (2000), "Jhon Dewey, una democracia vital”, en Pedagogías del siglo $x x$, Barcelona, Cisspraxis, s.A.

Bernstein, B. y Díaz, M. (1985), "Hacia una teoría del discurso pedagógico", en Revista Colombiana de Educación, núm. 15, Bogotá, Centro de Investigaciones (cIUP) Universidad Pedagógica Nacional.

- y Díaz, M. (1985), "Clases y pedagogías: Visibles e Invisibles", en Revista Colombiana de Educación, núm. 15, Bogotá, Centro de Investigaciones (CIUP) Universidad Pedagógica Nacional.

- (1993), Sociología de la Transmisión Cultural. Poder, Educación y Conciencia, Santiago de Chile, cIDE (reproducción realizada para funcionarios del SNP-ICFES V/97).

Betancourt, M.; Puche, M. E. (1997), El proyecto pedagógico, facilitador de un aprendizaje significativo, Bogotá, Ministerio de Educación Nacional, Serie Publicaciones para Maestros.

Castorina, J. A. (1998), Los problemas conceptuales del constructivismo y sus relaciones con la educación, en Debates constructivistas, capítulo I, Argentina, Aique.

Charaudeau P. y Dominique M. (2002), Diccionario de análisis del discurso, Madrid, Amorrortu.

Dewey, J. (1967), Democracia y educación. Una introducción a la filosofía de la educación, Buenos Aires, Losada.

- (1950), Las escuelas del mañana, Buenos Aires, Losada.

Fandiño, G. (1997), "El trabajo por proyectos y escuela nueva", en El proyecto pedagógico, facilitador de un aprendizaje significativo, Bogotá, Ministerio de Educación Nacional, Serie Publicaciones para Maestros.

Habermas, J. (1997), Conocimiento e Interés, Valencia, Educacion materials de Filosofia.

Hernández, F. y Ventura, M. (1996), La organización del curriculum por proyectos de trabajo. El conocimiento es un calidoscopio, Barcelona, 
Ice Institut de Ciéncies de 1' Educació \& GRAó de Serveis Pedagógics.

- (2002), "Los proyectos de trabajo: mapa para navegantes en mares de incertidumbre", en $\mathrm{Cua}^{-}$ dernos de Pedagogía, núm. 310, Barcelona,.

Jolibert, J. (1995), Formar niños productores de textos, Chile, Dolmen Ediciones.

- et al. (1999), Transformar la formación docente inicial. Propuesta en didáctica de Lengua Materna, Chile, Aula Xxi-Unesco-Santillana:

Jurado Valencia, (1999), Investigación, escritura y educación. El lenguaje y la literatura en la transformación de la escuela, Bogotá, Plaza \& Janés.

- (2003, abril-mayo), "La educación por proyectos: una pedagogía para la conjetura", en Revista Magisterio, núm. 002, Bogotá, Cooperativa Editorial Magisterio.

Kilpatrick, W.H., et al. (1944), El nuevo programa escolar, Buenos Aires, Losada.

Londoño Ramos, C. A. (2002), "El pragmatismo de Dewey y la escuela nueva en Colombia”, en Historia de la Educación Colombiana, Pereira, Doctorado en Ciencias de la Educación, Rudecolombia.

Luzuriaga, L. (1961), Métodos de la nueva educación, Buenos aires, Losada.

Mougniotte, A (1998). "Los métodos de enseñanza y de trabajo”, en Avanzini, G. (comp.), La pedagogía boy, México, Fondo de Cultura Económica.

Not, L. (2000), Las pedagogías del conocimiento, Colombia, Fondo de Cultura Económica.

- (1992), La enseñanza dialogante. Hacia una educación en segunda persona, Barcelona, Herder.

Rincón, G. (s.f.), El trabajo por proyectos y la enseñanza y el aprendizaje del lenguaje escrito en la educación primaria, Universidad del Valle, Escuela de Ciencias del Lenguaje.

Rodríguez (2001) "Antología de proyectos pedagógicos", en Cuadernos de Trabajo, núm. 2, Bogotá, Universidad Distrital Francisco José de Caldas.

Saint, F. (1961), "El método de proyectos", en Métodos de la nueva educación, Buenos Aires, Losada.
Torres, J. (1994), Globalización e interdisciplinariedad: El curriculum integrado, Barcelona, Morata.

Ventura, M. R. (2000), "Los proyectos de trabajo: Una forma de compartir preocupaciones”, en Cuadernos de Pedagogía, núm. 291.

\section{Documentos}

Especialización en Lenguaje y Pedagogía de Proyectos, (2001) "Antología de proyectos pedagógicos”, Cuadernos de Trabajo, núm. 2, Bogotá, Universidad Distrital Francisco José de Caldas.

Especialización en Lenguaje y Pedagogía de Proyectos (2001), La pedagogía de proyectos en acción. La ejecución de proyectos en las aulas de Bogotá. Sistematización de experiencias, Bogotá, Universidad Distrital Francisco José de Caldas.

Especialización en Lenguaje y Pedagogía de Proyectos (1999), La pedagogía de proyectos: opción de cambio social, Bogotá, Universidad Distrital Francisco José de Caldas, facultad de Ciencias y Educación.

Ministerio de Educación Nacional (1991), Propuesta curricular piloto para el grado cero: marco politico, conceptual y pedagógico, Bogotá.

- (1998), Lineamientos curriculares para el área de Lengua Castellana, Bogotá.

Programa de fortalecimiento de la capacidad científica en la educación básica y media (1997), Memorias del seminario El lenguaje en la ciencia y en la educación, Bogotá, Universidad Nacional de Colombia, Programa ReD.

- (1998), Memorias del seminario La investigación en la escuela, Bogotá, Universidad Nacional de Colombia, Programa RED.

Red Latinoamericana para la transformación de la formación docente en Lenguaje (2001), La formación docente en América Latina, Bogotá, Cooperativa editorial Magisterio.

Serie "Maestros Escribiendo 3" (1999), Proyectos con-sentidos, Programa de mejoramiento docente en lengua materna, Cali, Fondo Men-Icetex-Universidad del Valle-Escuela de Ciencias del Lenguaje. 


\begin{tabular}{|c|c|c|c|c|c|c|}
\hline \begin{tabular}{|c|}
$\begin{array}{c}\text { Denominación } \\
\text { y fuente }\end{array}$ \\
\end{tabular} & Concepto(s) & $\begin{array}{c}\text { Adscripción } \\
\text { teórica }\end{array}$ & Propósitos & Tipologías & $\begin{array}{c}\text { Ubicación en el } \\
\text { currículo }\end{array}$ & $\begin{array}{l}\text { Procedimientos } \\
\text { metodológicos }\end{array}$ \\
\hline $\begin{array}{l}\text { Trabajo por } \\
\text { Proyectos } \\
\text { Programa de } \\
\text { mejoramiento } \\
\text { docente } \\
\text { en Lengua } \\
\text { Materna } \\
\text { Universidad } \\
\text { del Valle }\end{array}$ & \begin{tabular}{|} 
Estrategia pedagógica \\
que implica un \\
proceso de planeación, \\
ejecución y evaluación \\
de manera completa, \\
intencional y \\
consciente. \\
Herramienta \\
cognitiva y \\
metacognitiva. \\
\end{tabular} & $\begin{array}{c}\text { Josette Jolibert } \\
\text { (1992) y } \\
\text { Fernando } \\
\text { Hernández } \\
\text { (1996). }\end{array}$ & $\begin{array}{c}\text { Integración } \\
\text { curricular. }\end{array}$ & $\begin{array}{l}\text { Proyectos } \\
\text { intraárea y } \\
\text { proyectos } \\
\text { interárea. }\end{array}$ & $\begin{array}{l}\text { Dentro del plan } \\
\text { de estudios, } \\
\text { tendiente a una } \\
\text { integración } \\
\text { curricular. }\end{array}$ & $\begin{array}{l}\text { Fase I: Planificación. } \\
\text { Fase II: Ejecución. } \\
\text { Fase III: Evaluación. }\end{array}$ \\
\hline $\begin{array}{l}\text { Pedagogía de } \\
\text { proyectos } \\
\text { Especialización } \\
\text { en Lenguaje y } \\
\text { Pedagogía de } \\
\text { Proyectos } \\
\text { Universidad } \\
\text { Distrital }\end{array}$ & $\begin{array}{l}\text { "Opción de cambio } \\
\text { en la cultura escolar". } \\
\text { "Concepción teórico- } \\
\text { metodológica flexible } \\
\text { y práctica que permite } \\
\text { alcanzar las metas } \\
\text { institucionales y } \\
\text { al mismo tiempo } \\
\text { dar respuesta a } \\
\text { las necesidades } \\
\text { individuales". }\end{array}$ & $\begin{array}{c}\text { Bricolaje de } \\
\text { autores y teorias: } \\
\text { Pedagogía' } \\
\text { de proyectos: } \\
\text { Dewey, Freinet, } \\
\text { Kilpatrick y } \\
\text { Vassileff, entre } \\
\text { otros. } \\
\text { Concepción } \\
\text { pedagógica: } \\
\text { Vygotsky, Bruner } \\
\text { y Coll, entre } \\
\text { otros. }\end{array}$ & \begin{tabular}{|c|} 
Desde el proyecto \\
del docente: \\
conocimiento \\
y comprensión \\
crítica de su labor \\
y del contexto \\
escolar a través de \\
la investigación- \\
acción en el aula. \\
\\
\\
Desde el proyecto \\
de aula: apropiación \\
de los saberes \\
por parte de los \\
estudiantes a través \\
de una dinámica \\
participativa y \\
de experiencias \\
significativas y \\
socializadoras.
\end{tabular} & $\begin{array}{c}\text { Proyecto } \\
\text { pedagógico } \\
\text { del } \\
\text { maestro. }\end{array}$ & $\begin{array}{c}\text { Dentro del plan } \\
\text { de estudios, } \\
\text { específicamente } \\
\text { en el área de } \\
\text { Lenguaje. }\end{array}$ & $\begin{array}{l}\text { Proyecto } \\
\text { pedagógico: } \\
\text { Formulación del } \\
\text { problema. } \\
\text { Recolección de la } \\
\text { información. } \\
\text { Análisis e } \\
\text { interpretación de los } \\
\text { datos. } \\
\text { Seguimiento y } \\
\text { evaluación. } \\
\\
\text { Proyecto de aula: } \\
\text { Fase I: planificación. } \\
\text { Fase II: Realización } \\
\text { de las tareas } \\
\text { de grupos o } \\
\text { individuales. } \\
\text { Fase III: Realización } \\
\text { terminal del objeto } \\
\text { del proyecto. } \\
\text { Fase IV: Evaluación } \\
\text { terminal del } \\
\text { proyecto. } \\
\text { Fase V: Evaluación } \\
\text { de los aprendizajes. }\end{array}$ \\
\hline $\begin{array}{l}\text { Proyectos de } \\
\text { aula } \\
\text { Programa RED } \\
\text { Universidad } \\
\text { Nacional }\end{array}$ & $\begin{array}{c}\text { Proceso de } \\
\text { investigación liderado } \\
\text { por el maestro en el } \\
\text { aula y al interior de } \\
\text { un campo específico } \\
\text { del saber. }\end{array}$ & $\begin{array}{l}\text { La adscripción } \\
\text { teórica depende } \\
\text { del objeto y } \\
\text { naturaleza de la } \\
\text { investigación. }\end{array}$ & $\begin{array}{l}\text { Adelantar procesos } \\
\text { de investigación } \\
\text { que transformen el } \\
\text { desempeño docente } \\
\text { y desarrollen la } \\
\text { capacidad científica } \\
\text { de la escuela. }\end{array}$ & $\begin{array}{l}\text { Proyecto } \\
\text { de aula. } \\
\text { Proyecto } \\
\text { de área. }\end{array}$ & $\begin{array}{l}\text { En un área } \\
\text { específica } \\
\text { dentro del plan } \\
\text { de estudios. }\end{array}$ & $\begin{array}{l}\text { Según el diseño } \\
\text { investigativo; } \\
\text { comprende } \\
\text { en general: } \\
\text { planteamiento } \\
\text { del problema, } \\
\text { justificación, } \\
\text { antecedentes, } \\
\text { marco teórico, } \\
\text { metodología, } \\
\text { resultados y } \\
\text { conclusiones. }\end{array}$ \\
\hline
\end{tabular}

Cuadro síntesis. Concepción de proyecto en los desarrollos teórico-prácticos en Colombia. 